\title{
INTEGRATED USE OF MANURE AND FERTILIZERS INCREASES RICE YIELD, NUTRIENT UPTAKE AND SOIL FERTILITY IN THE BORO-FALLOW-T.AMAN RICE CROPPING PATTERN
}

\author{
S. Bilkis ${ }^{1 *}$, M.R. Islam ${ }^{2}$, M. Jahiruddin ${ }^{2}$, M.M. Rahaman ${ }^{2}$ \\ ${ }^{1}$ Sylhet Agricultural University, Sylhet, Bangladesh \\ ${ }^{2}$ Department of Soil Science, Bangladesh Agricultural University, Mymensingh, Bangladesh
}

\begin{abstract}
The effect of integrated use of manure and fertilizers on crop yield, nutrient uptake and soil fertility was studied in the Boro-fallow-T. Aman cropping pattern over two years. The experiment was set up at Bangladesh Agricultural University (BAU) farm, Mymensingh under the AEZ 9 (Old Brahmaputra Floodplain). The field trial consisted of eight treatments and control (no fertilizer or manure), $100 \%$ chemical fertilizers (CF), and IPNS based six treatments with six types of manure. Cowdung (CD), CD slurry, Trichocompost (TC) and vermicompost (VC) were added to soil at $5 \mathrm{t} \mathrm{ha}^{-1}$ and poultry manure (PM) and PM slurry applied at $3 \mathrm{t} \mathrm{ha}^{-1}$. For all IPNS treatments, nutrient supply from manure was adjusted with that from chemical fertilizers. In each crop cycle, manure was applied to the first crop (Boro rice) and the residual effect was evaluated on the succeeding crop (T.Aman rice). The IPNS based treatments significantly increased the grain and straw yields of Boro rice and it had also positive residual effect on T.Aman rice. Trichocompost and vermicompost, among the six IPNS treatments, demonstrated higher crop yield and that was followed by poultry manure slurry and cowdung slurry. Integrated use of manure with fertilizers gave on an average $8.3-33.8 \%$ and $2.9-18.3 \%$ higher grain yield in Boro and $\mathrm{T}$. Aman rice, respectively over sole fertilizers treatment. Higher nutrient uptake by crops (N, P, K \& S) was also observed in IPNS treated plots. The IPNS treatments improved soil fertility in terms of increasing organic matter, N, P and S contents of soil after two crop cycles. The study suggests that manure and fertilizers should be used in an integrated manner to achieve sustainable crop yield, without incurring loss to soil fertility.
\end{abstract}

\footnotetext{
*Corresponding author e-mail: sbilkis26@gmail.com
} 
Keywords: Cowdung, poultry manure, trichocompost, vermicompost, rice yield, soil fertility

\section{INTRODUCTION}

In Bangladesh, with advancement of time, nutrient mining increases due to increasing cropping intensity (191\%, BBS 2017), use of modern varieties, nutrient leaching, gaseous loss, soil erosion and imbalanced application of fertilizers with no or little addition of organic manure. Higher is the crop yield, higher is the nutrient removal from soil. Nutrient deficiency in this country's soils has arisen chronologically $\mathrm{N}, \mathrm{P}, \mathrm{K}$, S, Zn and B (Islam, 2008; Jahiruddin and Satter, 2010). About 45\% of net cultivable areas of Bangladesh contain less than 1\% OM (FRG, 2012). Organic manure is a good source of nutrients, especially N, P \& S and it's a good means of soil rejuvenation (Jeptoo et al., 2013). So, use of OM could be an inevitable practice in the coming years for ensuring sustainable crop productivity without affecting soil fertility (Heikamp et al., 2011; Premsekhar and Rajashree, 2009).

Bio-slurry, by-product of biogas plant, can be a potential source of manure for crop production (Yu et al., 2010; Abubaker, 2012). Bio-slurry is the residual manure generated through anaerobic decomposition of various organic materials, chiefly cowdung and poultry manure, in presence of anaerobic microbes in the biogas digester. About $25-30 \%$ of organic matter is converted into biogas during the anaerobic fermentation process, while the rest becomes available as manure (bioslurry).

As stated by Rahman et al. (2008), unbalanced use of chemical fertilizers has affected soil health, causing a substantial decrease in soil organic carbon. As a general rule, use of organic fertilizers especially in composted form produces positive effect on soil health and fertility, which consequents increased crop yield on a long-term basis (Mehdizadeh et al., 2013).

Manure or fertilizer alone cannot sustain soil fertility and crop yield over time, their combination is essential (FRG, 2012). Nambiar (1997) viewed that integrated use of organic manure and chemical fertilizers would be quite promising not only in providing greater stability in production, but also in maintaining soil fertility status. Limited data are available on the growth, yield and nutrient content of agronomic crops treated with bioslurry, vermicompost and trichocompost. It is essential to develop a strong workable and compatible package of nutrient management through organic and inorganic sources for various crops based on scientific facts, local conditions and economic viability (Kannaiyan, 2000).

The present study was, therefore, undertaken to develop an efficient nutrient management practice following the concept of IPNS approach in the rice-rice cropping pattern. 


\section{MATERIALS AND METHODS}

The experiment was conducted at the soil science field laboratory of Bangladesh Agricultural University (BAU), Mymensingh for two consecutive years, 2012 and 2013. The experimental site was situated at $24.75^{\circ} \mathrm{N}$ latitude and $90.5^{\circ} \mathrm{E}$ longitude. The soil belongs to Sonatala series under agro-ecological zone (AEZ) 9, known as Old Brahmaputra Floodplain (FAO/UNDP, 1988). The soil was silt loam in texture having $6.29 \mathrm{pH}, 1.85 \% \mathrm{OM}, 0.124 \%$ total $\mathrm{N}, 3.96 \mathrm{mg} \mathrm{kg}^{-1}$ available $\mathrm{P}, 0.11 \mathrm{cmol} \mathrm{kg}^{-1}$ exchangeable $\mathrm{K}$ and $11.9 \mathrm{mg} \mathrm{kg}^{-1}$ available $\mathrm{S}$.

Variety BRRI dhan29 and BINA dhan 7 was used for Boro and T. Aman rice, respectively. The experiment was laid out in a randomized complete block design (RCBD), with three replications. There were eight treatments. as shown in table 1. Treatments $T_{3}-T_{8}$ received a definite amount of nutrients from different types of manure and the rest amount of nutrients came from chemical fertilizers so that the rate of every nutrient application was the same over the fertilizer and manure + fertilizer treatments. The fertilizer doses were rationalized for the second crop, as outlined in the Fertilizer Recommendation Guide (FRG, 2012). The rate of manure application was $5 \mathrm{t} \mathrm{ha}^{-1}$ for cowdung, its slurry, and tricho- and vermi compost, and 3 $\mathrm{t} \mathrm{ha}^{-1}$ for poultry manure and its slurry. Full amount of $\mathrm{P}, \mathrm{K}$ and $\mathrm{S}$ fertilizers was applied as basal during final land preparation and urea was applied in 3 equal splits the one-third during final land preparation and the two-thirds during tiller and panicle initiation stages of crop growth. All types of manure were applied seven days before transplanting. Thirty five days' old seedlings were used for transplanting of both Boro and T.Aman rice. Boro rice was transplanted on 24 January in 2012 and 28 January in 2013 and was harvested correspondingly on 20 May 2012 and 22 May 2013. T.Aman rice was transplanted on 6 August 2012 and 28 July 2013 and harvested respectively on 8 November 2012 and 9 November 2013. Weeding and irrigation were done whenever required.

At maturity, the crop was harvested and agronomic data viz. plant height, tillers hill ${ }^{-1}$, panicle length $(\mathrm{cm})$, grains panicle ${ }^{-1}, 1000$-grain weight $(\mathrm{g})$ and grain and straw yields were recorded. Grain yield was expressed at $14 \%$ moisture basis and straw yield at sun dry basis, drying was done for a period until a constant weight was obtained. The grain and straw samples were collected, dried and ground for analysis of $\mathrm{N}, \mathrm{P}, \mathrm{K} \& \mathrm{~S}$ contents following standard methods $\left(\mathrm{H}_{2} \mathrm{SO}_{4}\right.$ digestion for $\mathrm{N}$ and $\mathrm{HNO}_{3}-\mathrm{HClO}_{4}$ digestion for $\mathrm{P}, \mathrm{K} \& \mathrm{~S}$ ). Nutrient uptake by the grain and straw was calculated by multiplying their percent concentration with the corresponding yield. Composite soil samples were collected from every plot and prepared for chemical analysis. Soil $\mathrm{pH}$ was measured by glass electrode $\mathrm{pH}$ meter with soil-water ratio 1:2.5 (McLean, 1982), organic matter by wet oxidation method (Nelson and Sommers, 1982), N by Micro-Kjeldahl method (Bremner and Mulvaney, 1982), P by $0.5 \mathrm{M} \mathrm{NaHCO}$, pH 8.5 extraction method (Olsen and Sommers, 1982), K by NH4OAc, pH 7.0 extraction method (Barker and Surh, 1982), S by $0.15 \mathrm{M} \mathrm{CaCl}_{2}$ 
extraction method (Page et al., 1982), $\mathrm{Zn}$ by $0.005 \mathrm{M}$ DTPA, pH 7.3 extraction method (Lindsay and Norvell, 1978) and hot water-0.02M CaCl${ }_{2}$ method (Page et al., 1982). All data were analyzed statistically by MSTATc computer programme following the F-test and the mean comparisons of the treatments were done by Duncan's Multiple Range Test (DMRT) at 5\% level.

Table 1. Treatment combinations of organic manure and chemical fertilizers

\begin{tabular}{ll}
\hline Treatment code & Treatment combinations \\
\hline $\mathrm{T}_{1}$ & Control (no manure or fertilizer) \\
$\mathrm{T}_{2}$ & HYG based 100\% chemical fertilizer (CF) [FRG-2012] \\
$\mathrm{T}_{3}$ & $\mathrm{CD}+\mathrm{CF}$ (IPNS basis) \\
$\mathrm{T}_{4}$ & $\mathrm{CD}$ slurry + CF (IPNS basis) \\
$\mathrm{T}_{5}$ & $\mathrm{PM}+\mathrm{CF}$ (IPNS basis) \\
$\mathrm{T}_{6}$ & $\mathrm{PM}$ slurry + CF (IPNS basis) \\
$\mathrm{T}_{7}$ & $\mathrm{TC}+\mathrm{CF}$ (IPNS basis) \\
$\mathrm{T}_{8}$ & $\mathrm{VC}+\mathrm{CF}$ (IPNS basis) \\
\hline HYG = High Yield Goal, CF = Chemical Fertilizer, IPNS = Integrated Plant Nutrition System, CD = \\
Cowdung, CD slurry = Cowdung slurry, PM = Poultry manure, PM slurry = Poultry manure slurry, TC \\
$=$ Trichocompost, VC = Vermicompost
\end{tabular}

\section{RESULTS}

Effects of integrated use of manure and fertilizers were investigated directly on Boro rice and their residual effects on T.Aman rice. The results are described below.

\section{Yield and yield attributes of Boro rice}

Significant variation was recorded for the plant height of Boro rice (BRRI dhan29) due to application of different manure and chemical fertilizers (Table 1). The tallest plant $(101.5 \mathrm{~cm})$ was observed in $\mathrm{T}_{7}$ treatment (trichocompost) and the shortest (78.6 $\mathrm{cm})$ was found in control $\left(\mathrm{T}_{1}\right)$ plot. In the second year (2013), the $\mathrm{T}_{4}$ treatment recorded the highest plant height $(95.7 \mathrm{~cm})$, which was statistically similar with other treatments except control $\left(\mathrm{T}_{1}\right)$, the number of tillers hill ${ }^{-1}$ showed a significant variation due to imposed treatments (Table 2). The highest number of tillers hill ${ }^{-1}$ (14.8 in the first year and 12.3 in the second year) was found in $T_{7}$ treatment. The lowest number of tillers hill ${ }^{-1}$ was produced in $\mathrm{T}_{1}$ (control) treatment in both the years. Panicle length was not significantly influenced by the treatments (Table 2). In both years, the maximum number of grains panicle ${ }^{-1}$ (144.7 and 147.5) was recorded in $\mathrm{T}_{7}$ treatment. Manure treated plots produced higher number of grains panicle ${ }^{-1}$ as compared to absolute fertilizer treated plots. The highest 1000-grain weight was 
recorded in $\mathrm{T}_{7}$, which was statistically similar with all other treatments except control over the years.

The grain yield of Boro rice was significantly influenced by different treatments (Table 3). In 2012, the highest grain yield $\left(6.44 \mathrm{t} \mathrm{ha}^{-1}\right)$ was obtained from $\mathrm{T}_{7}$ treatment $(\mathrm{TC}+\mathrm{CF})$, which was statistically similar with $\mathrm{T}_{5}\left(6.20 \mathrm{t} \mathrm{ha}^{-1}\right), \mathrm{T}_{6}(6.30 \mathrm{t}$ $\left.\mathrm{ha}^{-1}\right)$ and $\mathrm{T}_{8}\left(6.34 \mathrm{t} \mathrm{ha}^{-1}\right)$. In this year all the manure treated plots produced higher yield compared to absolute chemical fertilizer $\left(\mathrm{T}_{2}\right)$ and control plots. Similar trend of grain yield was observed in 2013. In both years, the control treatment $\left(\mathrm{T}_{1}\right)$ produced the lowest grain yield. Like grain yield, the straw yield of Boro rice was markedly influenced by different treatments (Table 3). In 2012, the highest straw yield (6.79 t ha $^{-1}$ ) was obtained from $T_{7}$ treatment, which was statistically similar with $T_{4}, T_{5}, T_{6}$ and $\mathrm{T}_{8}$ treatments. In 2013, $\mathrm{T}_{7}$ recorded the highest straw yield $\left(7.48 \mathrm{t} \mathrm{ha}^{-1}\right)$, which was identical with all other treatments except $T_{2}$ and $T_{1}$. The lowest straw yields of $2.93 \mathrm{t} \mathrm{ha}^{-1}$ and $3.11 \mathrm{tha}^{-1}$ were recorded in control $\left(\mathrm{T}_{1}\right)$ treatment.

Table 2. Effects of integrated use of manure and fertilizers on plant height, tillers and panicle length of Boro rice (BRRI dhan29)

\begin{tabular}{l|lllll|l|l}
\hline \multirow{2}{*}{ Treatments } & \multicolumn{2}{|c|}{ Plant height $(\mathrm{cm})$} & \multicolumn{2}{c|}{ Tillers hill $^{-1}$} & \multicolumn{2}{c}{ Panicle length $(\mathrm{cm})$} \\
\cline { 2 - 7 } & 2012 & 2013 & 2012 & 2013 & 2012 & 2013 \\
\hline $\mathrm{T}_{1}$ & $78.6 \mathrm{~d}$ & $75.4 \mathrm{~b}$ & $8.8 \mathrm{c}$ & $6.2 \mathrm{~d}$ & $20.6 \mathrm{~b}$ & $22.2 \mathrm{c}$ \\
$\mathrm{T}_{2}$ & $94.7 \mathrm{c}$ & $92.6 \mathrm{a}$ & $13.0 \mathrm{~b}$ & $9.8 \mathrm{c}$ & $23.7 \mathrm{a}$ & $24.8 \mathrm{~b}$ \\
$\mathrm{~T}_{3}$ & $94.1 \mathrm{c}$ & $92.3 \mathrm{a}$ & $13.4 \mathrm{ab}$ & $9.7 \mathrm{c}$ & $23.7 \mathrm{a}$ & $25.2 \mathrm{ab}$ \\
$\mathrm{T}_{4}$ & $96.1 \mathrm{bc}$ & $95.7 \mathrm{a}$ & $14.3 \mathrm{ab}$ & $10.7 \mathrm{bc}$ & $24.3 \mathrm{a}$ & $25.8 \mathrm{ab}$ \\
$\mathrm{T}_{5}$ & $100.1 \mathrm{ab}$ & $95.5 \mathrm{a}$ & $14.8 \mathrm{a}$ & $11.2 \mathrm{ab}$ & $23.1 \mathrm{a}$ & $26.1 \mathrm{ab}$ \\
$\mathrm{T}_{6}$ & $96.9 \mathrm{abc}$ & $94.1 \mathrm{a}$ & $14.7 \mathrm{a}$ & $10.1 \mathrm{bc}$ & $23.8 \mathrm{a}$ & $26.6 \mathrm{ab}$ \\
$\mathrm{T}_{7}$ & $101.5 \mathrm{a}$ & $95.2 \mathrm{a}$ & $14.8 \mathrm{a}$ & $12.3 \mathrm{a}$ & $24.3 \mathrm{a}$ & $26.9 \mathrm{a}$ \\
$\mathrm{T}_{8}$ & $98.6 \mathrm{abc}$ & $95.1 \mathrm{a}$ & $14.8 \mathrm{a}$ & $11.1 \mathrm{~b}$ & $24.1 \mathrm{a}$ & $26.8 \mathrm{a}$ \\
\hline $\mathrm{CV}(\%)$ & 2.80 & 2.40 & 6.23 & 6.29 & 3.36 & 3.70 \\
Significant level & $* *$ & $* *$ & $* *$ & $* *$ & $* *$ & $* *$ \\
$\mathrm{SE}( \pm)$ & 1.536 & 1.274 & 0.519 & 0.368 & 0.454 & 0.547 \\
\hline
\end{tabular}

Means followed by same letter in a column are not significantly different at $5 \%$ level by DMRT.

$\mathrm{SE}( \pm)=$ standard error of means, $\mathrm{CV}=$ Coefficient of variation, ${ }^{* *}=$ Significant at $1 \%$ level 
Table 3. Effects of integrated use of manure and fertilizers on grains panicle ${ }^{-1}$ and 1000-grain weight and grain and straw yields of Boro rice (BRRI dhan29)

\begin{tabular}{|c|c|c|c|c|c|c|c|c|}
\hline \multirow[t]{2}{*}{ Treatments } & \multicolumn{2}{|c|}{ Grains panicle $^{-1}$} & \multicolumn{2}{|c|}{$\begin{array}{l}\text { 1000-grain weight } \\
\text { (g) }\end{array}$} & \multicolumn{2}{|c|}{ Grain yield $\left(\mathrm{t} \mathrm{ha}^{-1}\right)$} & \multicolumn{2}{|c|}{ Straw yield $\left(\mathrm{t} \mathrm{ha}^{-1}\right)$} \\
\hline & 2012 & 2013 & 2012 & 2013 & 2012 & 2013 & 2012 & 2013 \\
\hline $\mathrm{T} 1$ & $87.83 c$ & $93.83 \mathrm{c}$ & $19.15 b$ & $20.19 b$ & $2.61 \mathrm{c}$ & $2.71 \mathrm{c}$ & $2.93 \mathrm{c}$ & $3.11 \mathrm{c}$ \\
\hline $\mathrm{T} 2$ & $129.5 b$ & $135.4 \mathrm{~b}$ & $20.72 \mathrm{a}$ & $22.27 \mathrm{a}$ & $5.44 b$ & $5.96 \mathrm{~b}$ & $6.09 \mathrm{ab}$ & $6.17 \mathrm{~b}$ \\
\hline $\mathrm{T} 3$ & $135.3 \mathrm{ab}$ & $138.8 \mathrm{ab}$ & $20.70 \mathrm{a}$ & $22.37 \mathrm{a}$ & $5.49 \mathrm{~b}$ & $6.17 b$ & $6.04 b$ & $6.54 \mathrm{ab}$ \\
\hline $\mathrm{T} 4$ & $137.9 \mathrm{ab}$ & $141.2 \mathrm{ab}$ & $21.08 \mathrm{a}$ & $22.38 \mathrm{a}$ & $6.10 \mathrm{ab}$ & $6.54 \mathrm{ab}$ & $6.21 \mathrm{ab}$ & $6.69 \mathrm{ab}$ \\
\hline T5 & $137.5 \mathrm{ab}$ & $142.8 \mathrm{ab}$ & $21.08 \mathrm{a}$ & $22.40 \mathrm{a}$ & $6.20 \mathrm{a}$ & $6.77 \mathrm{ab}$ & $6.71 \mathrm{ab}$ & $7.22 \mathrm{a}$ \\
\hline T6 & $139.2 \mathrm{ab}$ & $144.5 \mathrm{ab}$ & $21.03 \mathrm{a}$ & $22.50 \mathrm{a}$ & $6.30 \mathrm{a}$ & $6.81 \mathrm{ab}$ & $6.43 \mathrm{ab}$ & $6.96 \mathrm{ab}$ \\
\hline $\mathrm{T} 7$ & $144.7 \mathrm{a}$ & $147.5 \mathrm{a}$ & $21.17 \mathrm{a}$ & $22.66 \mathrm{a}$ & $6.44 \mathrm{a}$ & $7.37 \mathrm{a}$ & $6.79 a$ & $7.48 \mathrm{a}$ \\
\hline $\mathrm{T} 8$ & $141.0 \mathrm{ab}$ & $146.3 \mathrm{a}$ & $21.07 \mathrm{a}$ & $22.53 \mathrm{a}$ & $6.34 \mathrm{a}$ & $7.31 \mathrm{a}$ & $6.63 \mathrm{ab}$ & $7.39 \mathrm{a}$ \\
\hline $\mathrm{CV}(\%)$ & 5.25 & 3.83 & 2.36 & 2.10 & 6.78 & 6.57 & 9.0 & 7.57 \\
\hline Sig. level & $* *$ & $* *$ & $* *$ & $* *$ & $* *$ & $* *$ & $* *$ & $* *$ \\
\hline $\mathrm{SE}( \pm)$ & 3.991 & 3.013 & 0.157 & 0.245 & 0.220 & 0.227 & 0.323 & 0.282 \\
\hline
\end{tabular}

Means followed by same letter in a column are not significantly different at $5 \%$ level by DMRT.

$\mathrm{SE}( \pm)=$ standard error of means, $\mathrm{CV}=$ Coefficient of variation, ${ }^{* *}=$ Significant at $1 \%$ level

\section{Nutrient uptake by Boro rice}

The $\mathrm{N}$ uptake by Boro rice was significantly affected by the treatments (Table 4). The highest total $\mathrm{N}$ uptake $\left(145.8 \mathrm{~kg} \mathrm{ha}^{-1}, 146.3 \mathrm{~kg} \mathrm{ha}^{-1}\right)$ was recorded in $\mathrm{T}_{7}$ treatment in both years. Cowdung, CD slurry, PM, PM slurry, trichocompost and vermicompost receiving treatments also showed higher $\mathrm{N}$ uptake in comparison to absolute chemical fertilizer $\left(\mathrm{T}_{2}\right)$ and control $\left(\mathrm{T}_{1}\right)$ treatments. The highest $\mathrm{K}$ uptake $(121.8 \mathrm{~kg}$ $\mathrm{ha}^{-1}$ ) in 2012 was recorded in $\mathrm{T}_{7}$ treatment, which was statistically similar with $\mathrm{T}_{8}$ $\left(120.7 \mathrm{~kg} \mathrm{ha}^{-1}\right)$ and $\mathrm{T}_{5}\left(116.8 \mathrm{~kg} \mathrm{ha}^{-1}\right)$. In 2013, the highest $\mathrm{K}$ uptake $\left(153.7 \mathrm{~kg} \mathrm{ha}^{-1}\right)$ was found in $\mathrm{T}_{7}$ and it was statistically similar with $\mathrm{T}_{4}\left(136.0 \mathrm{~kg} \mathrm{ha}^{-1}\right), \mathrm{T}_{5}(141.3 \mathrm{~kg}$ $\left.\mathrm{ha}^{-1}\right), \mathrm{T}_{6}\left(137.2 \mathrm{~kg} \mathrm{ha}^{-1}\right)$ and $\mathrm{T}_{8}\left(144.3 \mathrm{~kg} \mathrm{ha}^{-1}\right)$. To the lowest $\mathrm{K}$ uptake was always noted in control $\left(\mathrm{T}_{1}\right)$. For both years, the $\mathrm{T}_{7}$ showed the highest $\mathrm{P}$ uptake $(18.66 \mathrm{~kg}$ ha $^{-1}, 23.8 \mathrm{~kg} \mathrm{ha}^{-1}$ ), which was statistically similar with $\mathrm{T}_{5}, \mathrm{~T}_{6}$ and $\mathrm{T}_{8}$ treatments (Table 4). All other manure treated plots except $C D$ showed higher $P$ uptake as compared to $\mathrm{T}_{2}$ treatment. Application of manure and fertilizer significantly influenced $\mathrm{S}$ uptake by Boro rice (Table 4). The highest $S$ uptake was recorded from $T_{7}\left(12.08 \mathrm{~kg} \mathrm{ha}^{-1}\right.$, $11.73 \mathrm{~kg} \mathrm{ha}^{-1}$ ) and the lowest $\mathrm{S}$ uptake in $\mathrm{T}_{1}$ compared to $\mathrm{T}_{2}$ and other treatments. 
Table 4. Effects of integrated use of manure and fertilizers on the N, K, P and S uptake by Boro rice (BRRI dhan29)

\begin{tabular}{l|c|c|c|c|c|c|c|c}
\hline \multirow{2}{*}{ Treatments } & \multicolumn{2}{|c|}{$\mathrm{N}$ uptake $\left(\mathrm{kg} \mathrm{ha}^{-1}\right)$} & \multicolumn{2}{c|}{$\mathrm{K}$ uptake $\left(\mathrm{kg} \mathrm{ha}^{-1}\right)$} & \multicolumn{2}{c|}{$\mathrm{P}$ uptake $\left(\mathrm{kg} \mathrm{ha}^{-1}\right)$} & \multicolumn{2}{c}{$\mathrm{S}$ uptake $\left(\mathrm{kg} \mathrm{ha}^{-1}\right)$} \\
\cline { 2 - 9 } & 2012 & 2013 & 2012 & 2013 & 2012 & 2013 & 2012 & 2013 \\
\hline T1 & $43.26 \mathrm{~d}$ & $43.32 \mathrm{e}$ & $42.28 \mathrm{~d}$ & $53.48 \mathrm{~d}$ & $4.96 \mathrm{~d}$ & $6.94 \mathrm{~d}$ & $2.77 \mathrm{e}$ & $3.25 \mathrm{e}$ \\
T2 & $103.2 \mathrm{c}$ & $104.4 \mathrm{~d}$ & $105.2 \mathrm{bc}$ & $110.5 \mathrm{c}$ & $13.99 \mathrm{~b}$ & $17.04 \mathrm{c}$ & $9.64 \mathrm{~cd}$ & $8.94 \mathrm{~cd}$ \\
T3 & $99.04 \mathrm{c}$ & $109.5 \mathrm{~cd}$ & $103.9 \mathrm{bc}$ & $129.5 \mathrm{~b}$ & $11.93 \mathrm{c}$ & $18.04 \mathrm{c}$ & $9.51 \mathrm{~d}$ & $8.24 \mathrm{~d}$ \\
T4 & $121.7 \mathrm{~b}$ & $118.7 \mathrm{bc}$ & $100.4 \mathrm{c}$ & $136.0 \mathrm{ab}$ & $13.74 \mathrm{bc}$ & $18.00 \mathrm{c}$ & $9.56 \mathrm{~d}$ & $8.99 \mathrm{~cd}$ \\
T5 & $129.3 \mathrm{~b}$ & $118.9 \mathrm{bc}$ & $116.8 \mathrm{a}$ & $141.3 \mathrm{ab}$ & $18.39 \mathrm{a}$ & $21.67 \mathrm{ab}$ & $11.12 \mathrm{ab}$ & $10.11 \mathrm{bc}$ \\
T6 & $129.3 \mathrm{~b}$ & $125.9 \mathrm{~b}$ & $114.0 \mathrm{ab}$ & $137.2 \mathrm{ab}$ & $16.72 \mathrm{a}$ & $21.38 \mathrm{~b}$ & $10.12 \mathrm{bcd}$ & $9.95 \mathrm{bc}$ \\
T7 & $145.8 \mathrm{a}$ & $146.3 \mathrm{a}$ & $121.8 \mathrm{a}$ & $153.7 \mathrm{a}$ & $18.66 \mathrm{a}$ & $23.80 \mathrm{a}$ & $12.08 \mathrm{a}$ & $11.73 \mathrm{a}$ \\
T8 & $130.6 \mathrm{~b}$ & $132.5 \mathrm{~b}$ & $120.7 \mathrm{a}$ & $144.3 \mathrm{ab}$ & $17.15 \mathrm{a}$ & $22.47 \mathrm{ab}$ & $11.03 \mathrm{abc}$ & $10.35 \mathrm{~b}$ \\
CV $(\%)$ & 5.59 & 6.75 & 6.08 & 7.44 & 7.65 & 6.62 & 8.04 & 7.20 \\
Sig. level & $* *$ & $* *$ & $* *$ & $* *$ & $* *$ & $* *$ & $* *$ & $* *$ \\
SE ( $)$ & 3.638 & 4.384 & 3.618 & 5.401 & 0.638 & 0.713 & 0.440 & 0.372 \\
\hline
\end{tabular}

Means followed by same letter in a column are not significantly different at $5 \%$ level by DMRT.SE $( \pm)$ $=$ Standard error of means, $\mathrm{CV}=$ Coefficient of variation, $* *=$ Significant at $1 \%$ level

\section{Residual effects on T.Aman rice}

T.Aman rice received $\mathrm{N}, \mathrm{P}, \mathrm{K} \& \mathrm{~S}$ from chemical fertilizer source only. Plant height varied significantly due to residual effect of different types of manure used (Table 5). In the first year, $\mathrm{T}_{4}$ produced the tallest plant $(91.7 \mathrm{~cm})$ which was statistically similar with $T_{5}, T_{6}, T_{7}$ and $T_{8}$ treatments. In the second year, the tallest plants were observed with $\mathrm{T}_{8}$ treatment, which however was statistically identical with all other treatments except $\mathrm{T}_{1}$ which exhibited the shortest plants in both years. In 2012, $\mathrm{T}_{7}$ produced the maximum number of tillers hill ${ }^{-1}$ (Table 5) while in 2013 the highest number of tillers was found in $\mathrm{T}_{8}$ treatment. The minimum number of tillers hill ${ }^{-1}$ was always noted in the control $\left(\mathrm{T}_{1}\right)$.

In 2012, the highest panicle length $(26.8 \mathrm{~cm})$ was recorded in $\mathrm{T}_{7}$ and it was statistically similar with all other treatments except $\mathrm{T}_{1}$ and $\mathrm{T}_{2}$. In 2013, the treatment effects were mostly similar; both $\mathrm{T}_{7}$ and $\mathrm{T}_{8}$ produced the biggest size panicle (25.6 $\mathrm{cm})$. In 2012, the maximum number of grains panicle ${ }^{-1}$ was obtained from $\mathrm{T}_{7}$ treatment, which was statistically similar with $\mathrm{T}_{4}, \mathrm{~T}_{5}, \mathrm{~T}_{6}$ and $\mathrm{T}_{8}$ treatments (Table 6). Almost similar trends were observed in 2013. The 1000-grain weight ranged from $20.8-22.4 \mathrm{~g}$ in 2012 and $20.8-23.1 \mathrm{~g}$ in 2013 , the highest 1000 -grain weight being 
noted in $\mathrm{T}_{8}$ and $\mathrm{T}_{7}$, respectively (Table 6 ). The control treatment had the lowest 1000grain weight. However, in the first year, variation in 1000-grain weight due to different treatments was non-significant, and in the second year, all the treatments were similar except $\mathrm{T}_{3}$ and $\mathrm{T}_{1}$ in terms of 1000-grain weight.

Table 5. Residual effects of integrated use of manure and fertilizers on plant height, tiller production and panicle length of T.Aman rice (BINA dhan7)

\begin{tabular}{|c|c|c|c|c|c|c|}
\hline \multirow[t]{2}{*}{ Treatments } & \multicolumn{2}{|c|}{ Plant height $(\mathrm{cm})$} & \multicolumn{2}{|c|}{ Tillers hill ${ }^{-1}$} & \multicolumn{2}{|c|}{ Panicle length $(\mathrm{cm})$} \\
\hline & 2012 & 2013 & 2012 & 2013 & 2012 & 2013 \\
\hline $\mathrm{T} 1$ & $79.7 \mathrm{~d}$ & $80.5 b$ & $9.1 \mathrm{~d}$ & $8.4 \mathrm{c}$ & $21.96 c$ & $22.0 \mathrm{~b}$ \\
\hline $\mathrm{T} 2$ & $88.9 \mathrm{c}$ & $90.7 \mathrm{a}$ & $11.7 \mathrm{c}$ & $11.5 \mathrm{~b}$ & $25.68 b$ & $25.0 \mathrm{a}$ \\
\hline $\mathrm{T} 3$ & $89.5 b c$ & $91.8 \mathrm{a}$ & $11.8 \mathrm{bc}$ & $11.7 \mathrm{ab}$ & $25.73 \mathrm{ab}$ & $25.1 \mathrm{a}$ \\
\hline $\mathrm{T} 4$ & $91.7 \mathrm{a}$ & $90.4 \mathrm{a}$ & $11.8 \mathrm{bc}$ & $12.6 \mathrm{ab}$ & $26.03 \mathrm{ab}$ & $25.2 \mathrm{a}$ \\
\hline T5 & 89.9abc & $89.5 \mathrm{a}$ & 13.1abc & $12.7 \mathrm{ab}$ & 26.17ab & $25.3 \mathrm{a}$ \\
\hline T6 & $90.9 \mathrm{ab}$ & $90.9 \mathrm{a}$ & $12.5 \mathrm{abc}$ & $13.4 \mathrm{ab}$ & $26.11 \mathrm{ab}$ & $25.2 \mathrm{a}$ \\
\hline $\mathrm{T} 7$ & $91.6 \mathrm{a}$ & $92.1 \mathrm{a}$ & $13.9 \mathrm{a}$ & $13.9 \mathrm{a}$ & $26.82 \mathrm{a}$ & $25.6 \mathrm{a}$ \\
\hline $\mathrm{T} 8$ & 89.9abc & $92.5 \mathrm{a}$ & 13.2ab & $14.0 \mathrm{a}$ & $26.77 \mathrm{a}$ & $25.6 \mathrm{a}$ \\
\hline $\mathrm{CV}(\%)$ & 2.17 & 2.86 & 6.39 & 9.79 & 2.47 & 5.21 \\
\hline Significant level & $* *$ & $* *$ & $* *$ & $* *$ & $* *$ & $* *$ \\
\hline $\mathrm{SE}( \pm)$ & 0.576 & 1.106 & 0.447 & 0.694 & 0.323 & 0.675 \\
\hline
\end{tabular}

Means followed by same letter in a column are not significantly different at $5 \%$ level by DMRT.SE $( \pm)$ $=$ Standard error of means, CV $=$ Coefficient of variation, $* *=$ Significant at $1 \%$ level

The grain yield of T.Aman rice responded significantly to the residual effect of different treatments (Table 6). In 2012, the highest grain yield $\left(4.70 \mathrm{t} \mathrm{ha}^{-1}\right)$ was obtained in $\mathrm{T}_{7}$, and the next highest yield was due to $\mathrm{T}_{4}, \mathrm{~T}_{6}$ and $\mathrm{T}_{8}$ treatments, their yields were statistically similar. The lowest grain yield $\left(2.47 \mathrm{t} \mathrm{ha}^{-1}\right)$ was observed with the control $\left(\mathrm{T}_{1}\right)$. In 2013, $\mathrm{T}_{7}$ also produced the highest grain yield $\left(5.15 \mathrm{t} \mathrm{ha}^{-1}\right)$, which was statistically similar with $\mathrm{T}_{8}\left(4.78 \mathrm{t} \mathrm{ha}^{-1}\right)$. Combined application of manure with fertilizers gave better grain yield compared to sole chemical fertilizers. In 2012, the highest straw yield $\left(4.85 \mathrm{t} \mathrm{ha}^{-1}\right)$ was obtained from $\mathrm{T}_{7}$, which was identical with $\mathrm{T}_{8}$ (Table 6). In 2013, $\mathrm{T}_{7}$ gave the maximum straw yield $\left(5.03 \mathrm{t} \mathrm{ha}^{-1}\right)$, which was significantly higher than all other treatments. Considering mean yield of two years, $\mathrm{T}_{7}$ produced the highest straw yield $\left(4.94 \mathrm{t} \mathrm{ha}^{-1}\right)$. Other manure treatments exhibited comparable yields with $\mathrm{T}_{2}$. The lowest straw yield was recorded with the control $\left(\mathrm{T}_{1}\right)$.

\section{Nutrient uptake}

In 2012, the highest $\mathrm{N}$ uptake $\left(98.67 \mathrm{~kg} \mathrm{ha}^{-1}\right)$ was found in $\mathrm{T}_{7}$, which was similar to $\mathrm{T}_{8}$ (Table 7). Statistically similar $\mathrm{N}$ uptake was observed in $\mathrm{T}_{4}$ and $\mathrm{T}_{6}$ treatments, which was significantly higher than absolute fertilizer treatment $\left(\mathrm{T}_{2}\right)$. The lowest $\mathrm{N}$ 
uptake by rice $\left(37.72 \mathrm{~kg} \mathrm{ha}^{-1}\right)$ was noted in control $\left(\mathrm{T}_{1}\right)$. Similarly in 2013 , the higher $\mathrm{N}$ uptake $\left(99.47 \mathrm{~kg} \mathrm{ha}^{-1}\right)$ was recorded in $\mathrm{T}_{7}$ that was statistically similar with $\mathrm{T}_{8}$ $\left(91.61 \mathrm{~kg} \mathrm{ha}^{-1}\right)$.

Table 6. Effects of integrated use of manure and fertilizers on grains panicle ${ }^{-1}$ and 1000-grain weight, grain and straw yields of T. Aman rice (BINA dhan7)

\begin{tabular}{|c|c|c|c|c|c|c|c|c|}
\hline \multirow[t]{2}{*}{ Treatments } & \multicolumn{2}{|c|}{ Grains panicle $^{-1}$} & \multicolumn{2}{|c|}{$\begin{array}{l}\text { 1000-grain weight } \\
(\mathrm{g})\end{array}$} & \multicolumn{2}{|c|}{$\begin{array}{l}\text { Grain yield } \\
\left(\mathrm{t} \mathrm{ha}^{-1}\right)\end{array}$} & \multicolumn{2}{|c|}{$\begin{array}{l}\text { Straw yield } \\
\left(\mathrm{t} \mathrm{ha}^{-1}\right)\end{array}$} \\
\hline & 2012 & 2013 & 2012 & 2013 & 2012 & 2013 & 2012 & 2013 \\
\hline $\mathrm{T} 1$ & $78.5 \mathrm{c}$ & $72.5 \mathrm{c}$ & 20.81 & $20.82 c$ & $2.47 \mathrm{~d}$ & $2.60 \mathrm{e}$ & $2.83 \mathrm{e}$ & $2.66 \mathrm{~d}$ \\
\hline $\mathrm{T} 2$ & $100.5 b$ & $94.43 b$ & 21.93 & $22.49 \mathrm{ab}$ & $3.69 \mathrm{c}$ & $4.08 \mathrm{~d}$ & $3.81 \mathrm{~d}$ & $4.13 \mathrm{c}$ \\
\hline $\mathrm{T} 3$ & $101.2 b$ & $94.30 \mathrm{~b}$ & 21.74 & $22.44 b$ & $3.79 \mathrm{c}$ & $4.31 \mathrm{~cd}$ & $4.07 \mathrm{~cd}$ & $4.35 \mathrm{bc}$ \\
\hline $\mathrm{T} 4$ & $109.2 \mathrm{a}$ & $94.30 \mathrm{~b}$ & 22.11 & $22.63 \mathrm{ab}$ & $4.15 b$ & $4.38 \mathrm{bcd}$ & $4.34 b c$ & $4.45 b$ \\
\hline T5 & $110.8 \mathrm{a}$ & $100.0 \mathrm{ab}$ & 22.09 & $22.79 a b$ & $4.32 b$ & $4.41 \mathrm{bcd}$ & $4.35 \mathrm{bc}$ & $4.62 b$ \\
\hline T6 & $112.3 \mathrm{a}$ & $103.8 \mathrm{ab}$ & 22.04 & $22.78 \mathrm{ab}$ & $4.35 b$ & $4.58 \mathrm{bc}$ & $4.39 b c$ & $4.54 b$ \\
\hline $\mathrm{T} 7$ & $113.5 \mathrm{a}$ & $106.1 \mathrm{a}$ & 22.35 & $23.06 \mathrm{a}$ & $4.70 \mathrm{a}$ & $5.15 \mathrm{a}$ & $4.85 \mathrm{a}$ & $5.23 \mathrm{a}$ \\
\hline $\mathrm{T} 8$ & $112.8 \mathrm{a}$ & $103.9 \mathrm{ab}$ & 22.37 & $22.81 \mathrm{ab}$ & $4.35 b$ & $4.78 \mathrm{ab}$ & 4.64ab & $4.92 b$ \\
\hline $\mathrm{CV}(\%)$ & 3.67 & 5.46 & 2.99 & 3.55 & 4.65 & 5.88 & 5.96 & 3.56 \\
\hline Sig. level & $* *$ & $* *$ & NS & $* *$ & $* *$ & $* *$ & $* *$ & $* *$ \\
\hline $\operatorname{SE}( \pm)$ & 2.22 & 3.032 & 0.378 & 0.3314 & 0.106 & 0.145 & 0.149 & 0.876 \\
\hline
\end{tabular}

Means followed by same letter in a column are not significantly different at $5 \%$ level by DMRT.SE ( \pm ) $=$ Standard error of means, $\mathrm{CV}=$ Coefficient of variation, ${ }^{* *}=$ Significant at $1 \%$ level

There was a positive residual effect of the treatments on the K uptake by the crop. In 2012, the highest $\mathrm{K}$ uptake $\left(109.9 \mathrm{~kg} \mathrm{ha}^{-1}\right)$ was recorded with $\mathrm{T}_{7}$, which was statistically similar with $\mathrm{T}_{4}, \mathrm{~T}_{5}$ and $\mathrm{T}_{8}$ treatments $\left(97.96,100.7\right.$ and $100.1 \mathrm{~kg} \mathrm{ha}^{-1}$, respectively). The lowest $\mathrm{K}$ uptake by rice $\left(44.81 \mathrm{~kg} \mathrm{ha}^{-1}\right)$ was observed in control $\left(\mathrm{T}_{1}\right)$. In 2013 , the highest $\mathrm{K}$ uptake $\left(110.7 \mathrm{~kg} \mathrm{ha}^{-1}\right)$ was found in $\mathrm{T}_{7}$, which was statistically identical with $\mathrm{T}_{8}$. In 2012 , the highest $\mathrm{P}$ uptake $\left(14.12 \mathrm{~kg} \mathrm{ha}^{-1}\right)$ was recorded with $\mathrm{T}_{7}$ and it was statistically similar with $\mathrm{T}_{5}, \mathrm{~T}_{6}$ and $\mathrm{T}_{8}$ treatments, showing 13.18, 13.70 and $13.43 \mathrm{~kg} \mathrm{ha}^{-1}$, respectively. In 2013, the $\mathrm{T}_{7}$ also showed the highest $\mathrm{P}$ uptake $\left(15.84 \mathrm{~kg} \mathrm{ha}^{-1}\right)$, followed by $\mathrm{T}_{8}\left(15.13 \mathrm{~kg} \mathrm{ha}^{-1}\right)$. The $\mathrm{T}_{5}\left(14.03 \mathrm{~kg} \mathrm{ha}^{-}\right.$ $\left.{ }^{1}\right)$ and $\mathrm{T}_{6}\left(14.38 \mathrm{~kg} \mathrm{ha}^{-1}\right)$ had identical $\mathrm{P}$ uptake followed by the $\mathrm{T}_{3}$ and $\mathrm{T}_{4}$ treatments. The lowest $\mathrm{P}$ uptake by the crop $\left(6.09 \mathrm{~kg} \mathrm{ha}^{-1}\right.$ in 2012 and $6.61 \mathrm{~kg} \mathrm{ha}^{-1}$ in 2013) was observed in control $\left(\mathrm{T}_{1}\right)$ plot. In the first year, $\mathrm{T}_{7}$ treatment had the highest $(8.66 \mathrm{~kg}$ $\mathrm{ha}^{-1}$ ) S uptake, which was significantly different from all other treatments (Table 10). The lowest $\mathrm{S}$ uptake by the crop $\left(3.01 \mathrm{~kg} \mathrm{ha}^{-1}\right)$ was found in control $\left(\mathrm{T}_{1}\right)$ treatment. In the second year, the highest $\mathrm{S}$ uptake by T.Aman rice $\left(9.19 \mathrm{~kg} \mathrm{ha}^{-1}\right)$ was observed in $\mathrm{T}_{7}$ and the next highest $\mathrm{S}$ uptake $\left(8.37 \mathrm{~kg} \mathrm{ha}^{-1}\right)$ was noted in $\mathrm{T}_{8}$ treatment (Table 7). 
Table 7. Effects of integrated use of manure and fertilizers on N, K, P and S uptake by T.Aman rice (BINA dhan7)

\begin{tabular}{|c|c|c|c|c|c|c|c|c|}
\hline \multirow[t]{2}{*}{ Treatments } & \multicolumn{2}{|c|}{$\mathrm{N}$ uptake $\left(\mathrm{kg} \mathrm{ha}^{-1}\right)$} & \multicolumn{2}{|c|}{ K uptake $\left(\mathrm{kg} \mathrm{ha}^{-1}\right)$} & \multicolumn{2}{|c|}{ P uptake $\left(\mathrm{kg} \mathrm{ha}^{-1}\right)$} & \multicolumn{2}{|c|}{$\begin{array}{l}\text { S uptake (kg ha } \\
1 \text { ) }\end{array}$} \\
\hline & 2012 & 2013 & 2012 & 2013 & 2012 & 2013 & 2012 & 2013 \\
\hline $\mathrm{T} 1$ & $37.72 \mathrm{~d}$ & $41.63 \mathrm{e}$ & $44.81 d$ & $44.52 \mathrm{e}$ & $6.09 \mathrm{~d}$ & $6.61 \mathrm{e}$ & $3.01 \mathrm{e}$ & $3.88 \mathrm{~d}$ \\
\hline $\mathrm{T} 2$ & $62.10 \mathrm{~cd}$ & $73.47 \mathrm{~d}$ & $79.80 \mathrm{c}$ & $89.04 d$ & $10.74 \mathrm{c}$ & $12.22 \mathrm{~d}$ & $5.31 \mathrm{~d}$ & $6.71 \mathrm{c}$ \\
\hline $\mathrm{T} 3$ & $67.89 \mathrm{bcd}$ & $79.88 \mathrm{~cd}$ & $83.65 \mathrm{c}$ & $91.02 \mathrm{~cd}$ & $11.13 \mathrm{c}$ & $12.84 \mathrm{~cd}$ & $5.59 \mathrm{~d}$ & $6.99 \mathrm{c}$ \\
\hline $\mathrm{T} 4$ & $78.98 \mathrm{bc}$ & $84.58 b c$ & $97.96 \mathrm{ab}$ & $93.22 \mathrm{bcd}$ & $12.51 \mathrm{~b}$ & $12.98 \mathrm{~cd}$ & $6.52 \mathrm{c}$ & $7.11 \mathrm{c}$ \\
\hline T5 & $72.16 \mathrm{bcd}$ & $84.71 b c$ & $100.7 \mathrm{ab}$ & $97.31 b c$ & $13.18 \mathrm{ab}$ & $14.03 \mathrm{bc}$ & $5.89 \mathrm{~d}$ & $7.29 \mathrm{c}$ \\
\hline T6 & $74.64 b c$ & $87.24 \mathrm{bc}$ & $89.06 b c$ & $99.27 \mathrm{~b}$ & $13.70 \mathrm{ab}$ & $14.38 \mathrm{bc}$ & $7.72 b$ & $7.30 \mathrm{c}$ \\
\hline $\mathrm{T} 7$ & $98.67 \mathrm{a}$ & $99.47 \mathrm{a}$ & $109.9 a$ & $110.7 \mathrm{a}$ & $14.12 \mathrm{a}$ & $15.84 \mathrm{a}$ & $8.66 \mathrm{a}$ & $9.19 \mathrm{a}$ \\
\hline $\mathrm{T} 8$ & $84.49 \mathrm{ab}$ & $91.61 \mathrm{ab}$ & $100.1 \mathrm{ab}$ & $106.6 \mathrm{a}$ & $13.43 \mathrm{ab}$ & $15.13 \mathrm{ab}$ & $7.86 b$ & $8.37 \mathrm{~b}$ \\
\hline $\mathrm{CV}(\%)$ & 3.15 & 6.13 & 7.67 & 4.48 & 5.36 & 6.01 & 5.51 & 4.46 \\
\hline Sig. level & $* *$ & $* *$ & $* *$ & $* *$ & $* *$ & $* *$ & $* *$ & $* *$ \\
\hline $\mathrm{SE}( \pm)$ & 1.375 & 2.845 & 3.907 & 2.368 & 0.367 & 0.450 & 0.201 & 0.183 \\
\hline
\end{tabular}

Means followed by same letter in a column are not significantly different at $5 \%$ level by DMRT.SE $( \pm)$ $=$ Standard error of means, $\mathrm{CV}=$ Coefficient of variation, $* *=$ Significant at $1 \%$ level

\section{Change in soil properties}

Soil samples from every plot after two crop cycles were analyzed for $\mathrm{pH}$, organic matter, N, P, K, S, Zn and B contents (Table 8). There was a little change in soil pH and $\mathrm{OM}$ over the treatments. Total $\mathrm{N}$ content of soil was higher in plots when organic manure was added to soil and it ranged from $0.138-0.160 \%$ (initial level $0.132 \%$ ). There was a decreasing trend of $\mathrm{K}$ content in all the treatments compared to initial soil status. Available P content varied from 3.92 - $9.52 \mathrm{mg} \mathrm{kg}^{-1}$ (initial status $3.61 \mathrm{mg}$ $\mathrm{kg}^{-1}$ ). Addition of organic manure considerably increased the soil S level. The soil $\mathrm{Zn}$ content had remarkably increased, much increase being observed in the TC and PM slurry amended plots. Like $\mathrm{Zn}$ content, all the treatments except control increased the B content of soil. 
Table 8. Changes in soil properties as influenced by manure and fertilizer treatments in the Boro-fallow- T.Aman cropping pattern

\begin{tabular}{|c|c|c|c|c|c|c|c|c|}
\hline \multirow[t]{2}{*}{ Treatments } & \multirow[t]{2}{*}{$\mathrm{pH}$} & \multirow{2}{*}{$\begin{array}{l}\mathrm{OM} \\
(\%)\end{array}$} & \multirow[t]{2}{*}{$\begin{array}{c}\text { Total N } \\
(\%)\end{array}$} & \multirow{2}{*}{$\begin{array}{c}\mathrm{K} \\
(\mathrm{cmol} \\
\left.\mathrm{kg}^{-1}\right)\end{array}$} & $\mathrm{P}$ & $S$ & $\mathrm{Zn}$ & B \\
\hline & & & & & \multicolumn{4}{|c|}{$\left(\mathrm{mg} \mathrm{kg}^{-1}\right)$} \\
\hline Initial soil & 6.14 & 2.35 & 0.132 & 0.12 & 3.61 & 12.6 & 0.65 & 0.250 \\
\hline \multicolumn{9}{|c|}{ Post-harvest soil (after 2-crop cycle) } \\
\hline $\mathrm{T} 1$ & 5.99 & 2.34 & 0.138 & 0.087 & 3.92 & 11.17 & 0.63 & 0.231 \\
\hline $\mathrm{T} 2$ & 6.02 & 2.32 & 0.143 & 0.093 & 4.3 & 14.43 & 0.89 & 0.265 \\
\hline $\mathrm{T} 3$ & 6.16 & 2.55 & 0.154 & 0.106 & 4.77 & 14.63 & 0.87 & 0.461 \\
\hline $\mathrm{T} 4$ & 6.18 & 2.52 & 0.155 & 0.109 & 4.87 & 17.08 & 0.88 & 0.323 \\
\hline T5 & 6.18 & 2.54 & 0.157 & 0.113 & 7.60 & 18.91 & 0.91 & 0.431 \\
\hline T6 & 6.13 & 2.57 & 0.155 & 0.103 & 9.52 & 17.76 & 0.93 & 0.375 \\
\hline $\mathrm{T} 7$ & 6.31 & 2.48 & 0.159 & 0.118 & 7.38 & 18.53 & 0.95 & 0.415 \\
\hline $\mathrm{T} 8$ & 6.23 & 2.45 & 0.143 & 0.103 & 5.72 & 17.31 & 1.08 & 0.461 \\
\hline
\end{tabular}

\section{DISCUSSION}

Integrated use of inorganic and organic sources of nutrients (called IPNS) produced significantly higher grain and straw yields of both Boro and T.Aman rice compared to sole inorganic source (fertilizer) use. Treatment containing Trichocompost recorded the highest crop yield, followed by vermicompost, PM slurry and PM treatments. The trichocompost treated plots demonstrated $21.1 \%$ and $27.7 \%$ higher grain yield of Boro and T.Aman rice, respectively over $100 \%$ fertilizer treatment.

Use of Trichoderma compost technology is at initial level in our country. Trichoderma fungi decomposes organic materials in which they grow, colonize plant root system and attack other fungi in the plant's root system and releases compounds that activate plant defense mechanism (Rabbani, 2013). The potential of organic amendments over chemical fertilizers in suppression of disease incidence has long been recognized (Hadar, 2011; Pane et al., 2011; Bonanomi et al., 2007).

Zaman (2002) reported a comparable crop yield in the rice-rice pattern due to application of $70 \%$ NPKS fertilizers plus $3 \mathrm{t} \mathrm{ha}^{-1}$ poultry manure with $100 \%$ NPKS sole fertilizers. Rahman (2013) observed a 15-20\% yield increase due to combined use of fertilizers with poultry manure or household compost over $100 \%$ sole fertilizer treatment. Poultry manure can supply organic $\mathrm{C}$ to soil in one way and some growth hormones and concentrates feed to poultry birds can influence the plant growth on the other way.

Between slurry and original manure, the slurry treatment produced statistically higher yield of crops. As reported by Haque (2014), bio-slurry and original manure can give similar crop yield, with an advantage that cowdung slurry at $5 \mathrm{t} \mathrm{ha}^{-1}(15 \%$ moisture) 
application can substitute $18-32 \% \mathrm{~N}, 40-100 \% \mathrm{P}$ and $13-34 \% \mathrm{~K}$, and poultry manure slurry at $3 \mathrm{t} \mathrm{ha}^{-1}$ application can substitute $15-21 \% \mathrm{~N}, 50-100 \% \mathrm{P}$ and $8-18 \% \mathrm{~K}$ for the chemical fertilizers in the rice-based cropping systems.

Tricochompost, vermicompost, PM and PM slurry showed also higher and comparable N, P, K and S uptake. Organic manure releases nutrients slowly and it is reflected on the nutrient concentration as well as nutrient uptake, as reported by Saidu et al. (2012), Ayoola and Makinde (2007). The increased uptake of nutrients due to NPKS fertilization and organic manure application was due to addition of nutrients and proliferous root system developed under balanced nutrient application resulting in better absorption of water and nutrients along with improved physical environment (Laxminarayana, 2006; Kler and Walia, 2006).

Organic manure showed a positive effect on soil properties, as determined after two crop cycles. Soil organic matter and N contents, and P, S, Zn \& B availability in soil showed an increasing trend in IPNS treated plots. On the contrary, the exchangeable $\mathrm{K}$ content decreased across the treatments showing a K mining. Soil organic matter undergoes mineralization and releases substantial quantities of nitrogen, phosphorus, sulphur and smaller amount of micronutrients (Rahman et al., 2013). Vermicompost contains most nutrients in plant available form such as nitrate, phosphate and exchangeable calcium and soluble potassium (Edwards, 1998; Orozco et al., 1996). Organic fertilizer application, therefore exhibited potential in improving crop yield, $\mathrm{N}$ use efficiency and soil health in acid lateritic soil of the subtropical climate (Murmu et al., 2013).

Thus, sustainable production of crops cannot be achieved by using chemical fertilizers alone because of deterioration in soil physical and biological environments (Khan et al., 2008). Integrated use of both organic manure and chemical fertilizers appears as the best approach in providing greater stability in production and improving soil fertility status, as evidenced in the past (Islam et al., 2011; Sood, 2007; Singh and Lal, 2006).

\section{CONCLUSION}

Among the IPNS treatments, trichocompost and vermicompost containing treatments showed higher crop yield, followed by poultry manure slurry and cowdung slurry. The IPNS based treatments gave on an average 8.3-33.8\% and 2.9-18.3\% higher grain yield in Boro and T.Aman rice, respectively over sole chemical fertilizer treatment. Higher nutrient uptake by crops $(\mathrm{N}, \mathrm{K}, \mathrm{P} \& \mathrm{~S})$ was also observed in IPNS treated plots. The IPNS treatments improved soil fertility in terms of increasing organic matter, N, P and S contents of soil after two crop cycles. It is concluded that manure and fertilizers should be used in an integrated manner to achieve sustainable crop yield, with sustained soil fertility. 


\section{ACKNOWLEDGEMENT}

The work was supported by the Higher Education Quality Enhancement Project (HEQEP) which had provided full research cost and offered Ph.D. fellowship to the first author.

\section{REFERENCES}

Abubaker, J. (2012). Effects of fertilization with biogas residues on crop yield, soil microbiology and greenhouse gas emissions. Acta Universitatis Agriculturae Sueciae, Ph.D. Thesis, Dept. of Microbiology, Swedish University of Agricultural Sciences, 46, $1-79$.

Ayoola, O.T., and Makinde, E.A. (2007). Fertilizer treatment effects on performance of cassava under two planting patterns in a cassava- based cropping system in SouthWest Nigeria. Research Journal of Agriculture and Biological Sciences, 3(1), 13-20.

Barker, D.E., and Suhr, N.H. (1982). Potassium. Atomic absorption and flame emission spectroscopy. In: Methods of soil analysis, Part 2, Chemical and microbiological properties, A.L. Page, R.H. Miller and D.R. Keeney (eds.), p. 13-26. American Society of Agronomy Inc., Madison, WI, USA.

Bonanomi, G, Antignani, V., Pane, C., Scala, F. (2007). Suppression of soil borne fungal diseases with organic amendments. Journal of Plant Pathology, 89, 311-324.

Bremner, J.M., and Mulvaney, C.S. 1982. Total nitrogen. In: Methods of soil analysis, Part 2, Chemical and microbiological properties, A.L. Page, R.H. Miller and D. R. Keeney (eds.), p. 595-624. American Society of Agronomy and Soil Science Society of America, Inc., Madison, Wisconsin, USA.

BBS, (2017). Bangladesh Bureau of Statistics. The Year Book of Agricultural Statistics of Bangladesh. Stat. Div. Minis. Planning, Govt. Peoples Repub. Bangladesh, Dhaka.

Edwards, C.A. (1998). The use of earthworm in the breakdown and management of organic wastes. In Earthworm Ecology, C.A. Edwards (Ed) pp. 327-254, CRC Press Boco Roton, FL.

FAO/UNDP. (1988). Land Resources Appraisal of Bangladesh for Agricultural Development, Report-2, Agro-ecological Regions of Bangladesh, BGD/81/035.

FRG. (2012). Fertilizer Recommendation Guide. Bangladesh Agricultural Research Council (BARC), Dhaka, Bangladesh.

Hadar, Y. (2011). Suppressive compost: when plant pathology met microbial ecology, Phytoparasitica, 39, 311-314.

Haque, M.A. (2014). Mineralization of bioslurry and its integrated use with fertilizers in the rice based cropping systems. Ph. D Thesis, Department of Soil Science, Bangladesh Agricultural University, Mymensingh, Bangladesh.

Heitkamp, F., Raupp, J., and Ludwig, B. (2011). Soil organic matter pools and crop yields as affected by the rate of farmyard manure and use of biodynamic preparations in a sandy soil. Organic Agriculture. 11, 111-124. 
Islam, M.S. (2008). Soil fertility history, present status and future scenario in Bangladesh. Bangladesh Journal of Agriculture and Environment, 4,129 -152.

Islam, M.M., Karim, A.J.M.S., Jahiruddin, M., Majid, N.M., Miah, M.G., Ahmed, M.M., and Hakim, M.A. (2011). Effects of organic manure and chemical fertilizers on crops in the radish-stem amaranth-Indian spinach cropping pattern in homestead area. Australian Journal of Crop Science, 5, 1370-1378.

Jahiruddin, M., and Satter, M.A. (2010). Research priority in agriculture and development of vision document-2030 and beyond. Land and soil resource management. Bangladesh gricultural Research Council. Dhaka.

Kannaiyan. (2000). Bio-fertilizers - key factor in organic farming. The Hindu survey of Indian Agriculture, p. 165-173.

Khan, Z., Paigham, S., Muhammad, A., Zagda, K., Shah, P., and Arfi, M.(2000). Management of organic farming: effectiveness of farmyard manure and nitrogen for maize productivity. Sarhad Journal of Agriculture, 16(5), 461-465.

Jeptoo, A., Aguyoh, J. N., and Saidi, M. (2013). Improving Carrot Yield and Quality through the Use of Bio-Slurry Manure. Sustainable Agricultural Research, 2(1), 164-172.

Kler, D.S., and Walia, S.S. (2006). Organic, integrated and chemical farming in wheat under maize-wheat cropping system. Indian Journal of Agronomy, 51, 6-9.

Laxminarayana, K. (2006). Effect of integrated use of inorganic and organic manures on soil properties, yields and nutrient uptake of rice in Ultisols of Mizoram. Journal of the Indian Society of Soil Science, 54, 120-123.

Lindsay, W.L., and Norvell, W.A. (1978). Development of a DTPA soil test for Zn, Fe, Mn and Cu. Soil Science Society of America Journal, 42, 421-428.

McLean, E.O. (1982). Soil pH and lime requirement. In: Methods of soil analysis, Part 2, Chemical and microbiological properties, A.L. Page, R.H. Miller and D.R. Keeney (eds.), p. 199-224. American Society of Agronomy Inc., Madison, WI, USA.

Mehdizadeh, M., Dorbandi , E.I., Naseri-Rad, H., and Tobeh, A. (2013). Growth and yield of tomato (Lycopersicon esculentum Mill.) as influenced by different organic fertilizers. International Journal of Agronomy and Plant Production, 4(4), 734-738.

Murmu, K., Swain, D.K., and Ghosh, B.C. (2013). Comparative assessment of conventional and organic nutrient management on crop growth and yield and soil fertility in tomatosweet corn production system. Australian Journal of Crop Science, 7(11), 1617-1626.

Nambiar, K.K.M. (1997). Nutrient management for soil fertility restoration and sustaining cropping system productivity. In nutrient management practices in crops and cropping system. C.P. Ghonsikar and V.S. Shinde (eds.) Scientific Publishers. India. P. 213-250.

Nelson, D.W., and Sommers, L.E. (1982). Total carbon, organic carbon, and organic matter. In: Methods of soil analysis, Part 2, Chemical and microbiological properties, A.L. Page, R..H. Miller and D.R. Keeney (eds.), p.539-579. American Society of Agronomy Inc., Madison, WI, USA.

Olsen, S.R., and Sommers, L.E. (1982). Phosphorus. In: Methods of soil analysis, Part 2, Chemical and microbiological properties, A.L. Page, R.H. Miller and D.R. Keeney (eds.), p. 403-430. American Society of Agronomy Inc., Madison, WI, USA. 
Orozco, F.H., Cegarra, J., Trujillo, L.M., and Roig, A. (1996). Vermicomposting of coffee pulp using the earthworm Eisenia Foetida: effects on $\mathrm{C}$ and $\mathrm{N}$ contents and the availability of nutrients. Biology and Fertility of Soil, 22, 162-166.

Page, A.L., Miller, R.H., and Keeney, D.R. (1982). Methods of soil analysis part $21^{\text {th }}$ edn. Amer. Soc. Agron. Inc. Madi Wisconsis, USA.

Pane, C., Spaccini, R., Piccolo, A., Scala, F., and Bonanomi, G. (2011). Compost amendments enhance peat suppressiveness to Pythium ultimum, Rhizoctonia solani and Sclerotinia minor. Biological Control, 56, 115-124.

Premsekhar, M., and Rajashree, V. (2009). Influence Organic Manures on Growth, Yield and Quality of Okra. . American-Eurasian Journal of Sustainable Agriculture, 3(1), 6-8.

Rabbani, M.G., Jahiruddin, M., Islam, M.S., and Haque, M.M.(2013). Assessment of the Fertilizer Market with Focus on Micronutrient and Organic Compost. Study Report, KATALYST, Dhaka.

Rahman, M.A. (2013). Integrated use of fertilizer with manure on mustard, potato and wheat and their residual effects on succeeding crops. Ph. D. Thesis. Department of Soil Science, Bangladesh Agricultural University, Mymensingh.

Rahman, M.H., Islam, M.R., Jahiruddin, M., Rafii, M.Y., Hanafi, M.M., and Malek, M.A. (2013). Integrated nutrient management in maize-legume-rice cropping pattern and its impact on soil fertility. Journal of Food, Agriculture \& Environment, 11(1), 648-652.

Rahman, S.M.E., Islam, M.A., Rahman, M.M., and Oh, D.H. (2008). Effect of cattle slurry on growth, biomass yield and chemical composition of maize fodder. Asian-Australian Journal of Animal Science, 2 (1), 1592-1598.

Saidu, A., Abayomi, Yakeen, A., Aduloju., and Morolake, O.(2012). Evaluation of complementry use of organic and inorganic fertilizers on the performance of upland rice (Oryza sativa L.). International Journal of Advanced Biological Research, 2(3), 487-491.

Singh, S.K., and Lal, S.S. (2006). Effect of organic sources of nutrients on potato production in South Bihar. Paper presented at national symposium on conservation agriculture and environment, BHU, Varanasi, India, 26-28 October 2006, 284.

Sood, M.C. (2007). Integrated nutrient supply and management for potato production in Mid Hills of Shimla. Potato Journal, 34, 101-102.

Yu, F.B., Luo, X.P., Song, C.F., Zhang, M.X., and Shan, S.D. (2010). Concentrated biogas slurry enhanced soil fertility and tomato quality. Plant Soil Science, 60(3), 262-268.

Zaman, S.K. (2002). Integration of fertilizer and manure for susainable soil fertility and productivity in rice-rice cropping pattern. Ph.D. Thesis. Dept. Soil Sci., BAU, Mymensingh. 